\title{
Existence of Sign-Changing Solution with Least Energy for a Class of Schrödinger-Poisson Equations in $\mathbb{R}^{3}$
}

\author{
Yaolan Tang, Qiongfen Zhang* (D) \\ College of Science, Guilin University of Technology, Guilin, China \\ Email: *qfzhangcsu@163.com
}

How to cite this paper: Tang, Y.L. and Zhang, Q.F. (2021) Existence of SignChanging Solution with Least Energy for a Class of Schrödinger-Poisson Equations in $\mathbb{R}^{3}$. Journal of Applied Mathematics and Physics, 9, 2483-2499.

https://doi.org/10.4236/jamp.2021.910159

Received: September 9, 2021

Accepted: October 18, 2021

Published: October 21, 2021

Copyright $\odot 2021$ by author(s) and Scientific Research Publishing Inc. This work is licensed under the Creative Commons Attribution International License (CC BY 4.0).

http://creativecommons.org/licenses/by/4.0/

\begin{abstract}
The nodal solutions of equations are considered to be more difficult than the positive solutions and the ground state solutions. Based on this, this paper intends to study nodal solutions for a kind of Schrödinger-Poisson equation. We consider a class of Schrödinger-Poisson equation with variable potential under weaker conditions in this paper. By introducing some new techniques and using truncated functional, Hardy inequality and Pohožaev identity, we obtain an existence result of a least energy sign-changing solution and a ground state solution for this kind of Schrödinger-Poisson equation. Moreover, the energy of the sign-changing solution is strictly greater than the ground state energy.
\end{abstract}

\section{Keywords}

Schrödinger-Poisson System, Sign-Changing Solution, Ground State Solution, Pohožaev Identity

\section{Introduction}

In this paper, the following nonlinear Schrödinger-Poisson system will be discussed

$$
\begin{cases}-\Delta u+V(x) u+\lambda \phi(x) u=f(u), & x \in \mathbb{R}^{3}, \\ -\Delta \phi=u^{2}, & x \in \mathbb{R}^{3},\end{cases}
$$

where the potential function $V: \mathbb{R}^{3} \rightarrow \mathbb{R}, \lambda>0$ is a parameter and $f \in C\left(\mathbb{R}^{3}, \mathbb{R}\right)$. We can assume that $f$ satisfies the following assumptions:

(f1) $\lim _{t \rightarrow 0} \frac{f(t)}{t}=0$; 
(f2) $\lim _{t \rightarrow \infty} \frac{f(t)}{t^{5}}=0$;

(f3) $\lim _{t \rightarrow+\infty} \frac{F(t)}{t^{2}}=+\infty$, where $F(t)=\int_{0}^{t} f(s) \mathrm{d} s$;

(f4) $\frac{f(t)}{|t|}$ is an increasing function of $\mathbb{R} \backslash\{0\}$.

To avoid involving too munch details for checking the compactness, we may assume that $V \in C\left(\mathbb{R}^{3},[0,+\infty)\right)$ and satisfies:

(V1) $V_{0} \leq V(x) \leq V_{\infty}:=\lim _{|y| \rightarrow \infty} V(y)$ for all $x \in \mathbb{R}^{3}$, where $V_{0}$ is a positive constant; meanwhile, we set up the weak decay hypothesis on $\nabla V$ :

(V2) there is $\theta \in[0,1)$ such that $\nabla V(x) \cdot x \leq \frac{\theta}{2|x|^{2}}, x \in \mathbb{R}^{3} \backslash\{0\}$.

We could also call system (1.1) as Schrödinger-Maxwell system, which is used in physics. In fact, the coupled nonlinear Schrödinger equation and Poisson equation can be used to describe the interaction of charged particles with electromagnetic fields. To learn more about the physical aspects of the Schrödinger-Poisson equation, the reader can read the related literature [1] [2] [3] and the references therein. What's more, readers can also read the following articles, including [4] [5] [6], which show the mathematical and physical background of system (1.1).

In recent years, there has been a lot of research on the solutions of Schrödinger-Poisson equation, especially the existence of positive solutions, multiple solutions, sign-changing solutions, ground state solutions and semi-classical states, we can look at literatures [2] [5] [7]-[14] and references therein. In addition, the research on the existence of sign-changing solutions is in [15]-[20], etc.

As we can see, Wang and Shuai in [17] also studied problem (1.1) and they obtained the existence of sign-changing solution to problem (1.1). They assumed that $V \in C\left(\mathbb{R}^{3}, \mathbb{R}\right)$ and $f \in C^{1}(\mathbb{R})$ satisfies (f1), (f2) and the following conditions:

(f3)' $\lim _{t \rightarrow \infty} \frac{F(t)}{t^{4}}=+\infty ;$

(f4), $\frac{f(t)}{|t|^{3}}$ is an increasing function of $\mathbb{R} \backslash\{0\}$.

By introducing a parameter $\mu \in[0,1]$, they show that any sign-changing solution for system (1.1) is strictly greater than twice the least energy solution. What's more, they combine the constrained variational method with the quantitative deformation lemma to prove the existence of the least energy signchanging solution. In addition, the energy doubling and asymptotic properties of the solution are also discussed. In contrast to Wang and Shuai's proof, we refer to the truncation function, which is inspired by [21] [22] [23] [24].

In [13], the following system is considered

$$
\left\{\begin{array}{l}
-\Delta u+u+\lambda \phi u=u^{p}, \\
-\Delta \phi=u^{2}, \lim _{|x| \rightarrow+\infty} \phi(x)=0,
\end{array}\right.
$$


where $V \equiv 1, f(u)=u^{p}$ and $1<p<5$. The authors obtained some existence and nonexistence results of positive radial solutions by using variational method, depending on the parameters $\lambda$ and $p$. It turns out that $p=2$ is the critical value for the existence and nonexistence of solutions. However, their study of the existence of positive radial solutions for system (1.2) is dependent on the parameter $\lambda>0$, which seems difficult to be applied to similar systems with variable potential.

Zhang in [22] consider the following Schrödinger-Poisson equation

$$
\begin{cases}-\Delta u+V_{1} u+\mu \phi u=f(u), & \text { in } \mathbb{R}^{3}, \\ -\Delta \phi=\mu u^{2}, & \text { in } \mathbb{R}^{3},\end{cases}
$$

where $V_{1}, \mu>0, f$ has a critical growth. The author obtained the existence of solutions for system (1.3) with a general nonlinearity in the critical growth by variational method. But he did not study the existence of sign-changing.

Sofiane Khoutir in [25] considered the following system

$$
\begin{cases}-\Delta u+V_{2} u+\lambda \phi u=f(u), & x \in \mathbb{R}^{3}, \\ -\Delta \phi=u^{2}, & x \in \mathbb{R}^{3},\end{cases}
$$

where $V_{2}$ is a positive constant. By using variational methods in combination with the Pohožaev identity, Sofiane Khoutir proved that system (1.4) has the least energy sign-changing solution and a ground state solution provided that $\lambda$ is sufficiently small. However, if the potential is not a positive constant, for example, the potential is variable, that is $V(x)$, it is very difficult to verify the Sobolev embedding compactness.

In our work, we consider variable potential $V(x)$ and put some constraints on it, and then study the least energy sign-changing solution and ground state solution of the Schrödinger-Poisson Equation (1.1).

We now need to introduce some symbolic notations. As usual, for $1 \leq p<+\infty$, let

$$
\|u\|_{p}^{p}:=\int_{\mathbb{R}^{3}}|u|^{p} \mathrm{~d} x, \quad u \in L^{p}\left(\mathbb{R}^{3}\right) .
$$

Let

$$
H^{1}\left(\mathbb{R}^{3}\right)=\left\{u \in L^{2}\left(\mathbb{R}^{3}\right): \int_{\mathbb{R}^{3}}\left(|\nabla u|^{2}+V(x) u^{2}\right) \mathrm{d} x<+\infty\right\},
$$

with the inner product and norm

$$
\langle u, v\rangle=\int_{\mathbb{R}^{3}}(\nabla u \nabla v+V(x) u v) \mathrm{d} x, \quad\|u\|^{2}=\int_{\mathbb{R}^{3}}\left(|\nabla u|^{2}+V(x) u^{2}\right) \mathrm{d} x .
$$

Therefore, the embedding $H^{1}\left(\mathbb{R}^{3}\right) \hookrightarrow L^{p}\left(\mathbb{R}^{3}\right)$ is continuous for $p \in[2,6]$, moreover, there exists a constant $C_{p}^{\prime}>0$ such that

$$
\|u\|_{p} \leq C_{p}^{\prime}\|u\|, \quad u \in H^{1}\left(\mathbb{R}^{3}\right), \quad p \in[2,6] .
$$

Let

$$
H:=H_{r}^{1}\left(\mathbb{R}^{3}\right)=\left\{u \in H^{1}\left(\mathbb{R}^{3}\right): u(x)=u(|x|)\right\} .
$$

Then, $H \subset H^{1}\left(\mathbb{R}^{3}\right)$, for $2<p<6$, the embedding $H \hookrightarrow L^{p}\left(\mathbb{R}^{3}\right)$ is 
compact.

Let $\mathcal{D}^{1,2}\left(\mathbb{R}^{3}\right):=\left\{u \in L^{6}\left(\mathbb{R}^{3}\right): \nabla u \in L^{2}\left(\mathbb{R}^{3}\right)\right\}$ be the Sobolev space with norm

$$
\|u\|_{\mathcal{D}^{1,2}}^{2}=\int_{\mathbb{R}^{3}}|\nabla u|^{2} \mathrm{~d} x \text {. }
$$

Then, the embedding $\mathcal{D}^{1,2}\left(\mathbb{R}^{3}\right) \hookrightarrow L^{6}\left(\mathbb{R}^{3}\right)$ is continuous (see [26]) and the best Sobolev constant is

$$
S:=\inf _{u \in \mathcal{D}^{1,2}\left(\mathbb{R}^{3}\right) \backslash\{0\}} \frac{\int_{\mathbb{R}^{3}}|\nabla u|^{2} \mathrm{~d} x}{\left(\int_{\mathbb{R}^{3}}|u|^{6} \mathrm{~d} x\right)^{\frac{1}{3}}} .
$$

We have known that for any $u \in H^{1}\left(\mathbb{R}^{3}\right)$, if $\phi_{u}$ is the unique solution of $-\Delta \phi=u^{2}$ in $\mathcal{D}^{1,2}\left(\mathbb{R}^{3}\right)$, then

$$
\phi_{u}(x)=\frac{1}{4 \pi} \int_{\mathbb{R}^{3}} \frac{u^{2}(y)}{|x-y|} \mathrm{d} x .
$$

What's more, the properties of $\phi_{u}$ are as follows (the detail proof can be seen in [27]):

Lemma 1.1. For $u \in H^{1}\left(\mathbb{R}^{3}\right)$, we have

(i) $\phi_{u} \geq 0, \forall u \in H^{1}\left(\mathbb{R}^{3}\right)$;

(ii) $\phi_{t}(u)=t^{2} \phi_{u}, \forall t>0, \forall u \in H^{1}\left(\mathbb{R}^{3}\right)$;

(iii) If $u_{n} \rightarrow u$ weakly in $H^{1}\left(\mathbb{R}^{3}\right)$, then $\phi_{u_{n}} \rightarrow \phi_{u}$ weakly in $\mathcal{D}^{1,2}\left(\mathbb{R}^{3}\right)$ and

$$
\int_{\mathbb{R}^{3}} \phi_{u} u^{2} \mathrm{~d} x \leq \liminf _{n \rightarrow \infty} \int_{\mathbb{R}^{3}} \phi_{u_{n}} u_{n}^{2} \mathrm{~d} x ;
$$

(iv) There exists a constant $C_{1}>0$, by Hölder inequality, such that

$$
\left\|\phi_{u}\right\|_{\mathcal{D}^{1,2}}^{2}=\int_{\mathbb{R}^{3}} \phi_{u} u^{2} \mathrm{~d} x \leq\left(\int_{\mathbb{R}^{3}} \phi_{u}^{2} \mathrm{~d} x\right)^{\frac{1}{2}}\left(\int_{\mathbb{R}^{3}} u^{4} \mathrm{~d} x\right)^{\frac{1}{2}}:=C_{1}\|u\|^{4}, \forall u \in H ;
$$

(v) If $u$ is a radial function, then $\phi_{u}$ is radial.

Now, we consider a family of $K_{\lambda}: H \rightarrow \mathbb{R}$ defined by

$$
K_{\lambda}(u)=\frac{1}{2} \int_{\mathbb{R}^{3}}\left(|\nabla u|^{2}+V(x) u^{2}\right) \mathrm{d} x+\frac{\lambda}{4} \int_{\mathbb{R}^{3}} \phi_{u} u^{2} \mathrm{~d} x-\int_{\mathbb{R}^{3}} F(u) \mathrm{d} x .
$$

Hence, by (f1), (f2), (V1) and (V2), $K_{\lambda}$ is well defined and $K_{\lambda} \in C^{1}(H, \mathbb{R})$. For any $u, v \in H$, there is

$$
\left\langle K_{\lambda}^{\prime}(u), v\right\rangle=\int_{\mathbb{R}^{3}}[\nabla u \nabla v+V(x) u v] \mathrm{d} x+\lambda \int_{\mathbb{R}^{3}} \phi_{u} u v \mathrm{~d} x-\int_{\mathbb{R}^{3}} f(u) v \mathrm{~d} x .
$$

Note that $\left(u, \phi_{u}\right) \in H^{1}\left(\mathbb{R}^{3}\right) \times \mathcal{D}^{1,2}\left(\mathbb{R}^{3}\right)$ is a solution of problem (1.1) if and only if $u \in H^{1}\left(\mathbb{R}^{3}\right)$ is a critical point of $K_{\lambda}$ and $\phi=\phi_{u}$. Moreover, the critical points of $K_{\lambda}$ on $H$ are the critical points of $K_{\lambda}$ on $H^{1}\left(\mathbb{R}^{3}\right)$ by the critical principle of symmetry. So, finding the weak solution of problem (1.1) is equivalent to finding the critical point of the functional $K_{\lambda}$.

In this paper, we denote

$$
u^{+}=\max \{u(x), 0\} \quad \text { and } \quad u^{-}=\min \{u(x), 0\},
$$

then $u=u^{+}+u^{-}$. 
We define the Nehari manifold for the energy functional $K_{\lambda}$ of problem (1.1) as

$$
\mathcal{N}_{\lambda}=\left\{u \in H \backslash\{0\},\left\langle K_{\lambda}^{\prime}(u), u\right\rangle=0\right\},
$$

and the nodal-Nehari manifold

$$
\mathcal{M}_{\lambda}=\left\{u \in H, u^{ \pm} \neq 0 \text { and }\left\langle K_{\lambda}^{\prime}(u), u^{ \pm}\right\rangle=0\right\} .
$$

What's more, we denote

$$
c_{\lambda}:=\inf _{u \in \mathcal{N}_{\lambda}} K_{\lambda}(u) \text { and } g_{\lambda}:=\inf _{u \in \mathcal{M}_{\lambda}} K_{\lambda}(u) .
$$

Moreover, $C_{1}, C_{2}, \cdots$ denote positive constants possibly different in different places. Strong convergence is expressed in terms of $\rightarrow$ and weak convergence is expressed in terms of $\rightarrow$.

The main result of this paper is presented as follows.

Theorem 1.1. Assume that (f1)-(f4), (V1) and (V2) hold. Then there exists a positive $\Upsilon$ such that for all $\lambda \in(0, \Upsilon)$, problem (1.1) has a least energy signchanging solution $z_{\lambda} \in \mathcal{M}_{\lambda}$ and a ground solution $u_{\lambda} \in \mathcal{N}_{\lambda}$ which is constant sign. In addition, these two solutions satisfy the following relationship

$$
g_{\lambda}=K_{\lambda}\left(z_{\lambda}\right)>K_{\lambda}\left(u_{\lambda}\right)=c_{\lambda} .
$$

Remark 1.1. It is easy to see that (f3) and (f4) are weaker than (f3)' and (f4)', respectively, so our result can be seen as a generalization of the result in [17]. Besides, we consider variable potential, from this point, our result can be seen as a slight generalization and improvement of [25].

The paper is organized as follows. In Section 2, we provide some lemmas, which are crucial to prove the main result of this paper. Section 3 is devoted to the proof of Theorem 1.1.

\section{Preliminaries}

We shall obtain a critical point of $t_{\lambda}$ by a mountain pass type argument, however, even though it is likely that critical point has a mountain pass geometry, showing that the (PS) sequence at the mountain-pass level are bounded seems out of reach under our weak assumptions on $f$. To overcome this difficulty, inspired by [21] [22] [23] [24], which consists in truncating the "rest" term of $t_{\lambda}$ outside of a ball centered at the origin and to show that, as $\lambda>0$ goes to zero, all (PS) sequences at the mountain-pass level lie in this ball, which is called truncated technique. Precisely, let $T>0$ be the truncation radius and consider a smooth function $\eta \in C^{1}\left(\mathbb{R}^{+}, \mathbb{R}\right)$ satisfying $0 \leq \eta(t) \leq 1,\left\|\eta^{\prime}\right\|_{\infty} \leq 2$,

$$
\eta(t)= \begin{cases}1, & t \in[0,1], \\ 0, & t \in[2, \infty),\end{cases}
$$

and $\eta$ is not increasing on [1,2]. Similar to [21] [22] [23] [24], for any positive constant $T>0$, we consider the truncated functional $K_{\lambda, T}: H \rightarrow \mathbb{R}$ defined by 


$$
K_{\lambda, T}(u)=\frac{1}{2}\|u\|^{2}+\frac{\lambda}{4} D_{T}(u) \int_{\mathbb{R}^{3}} \phi_{u} u^{2} \mathrm{~d} x-\int_{\mathbb{R}^{3}} F(u) \mathrm{d} x,
$$

where $D_{T}(u)=\eta\left(\frac{\|u\|^{2}}{T^{2}}\right)$. From (f1), (f2), (V1) and (V2), it is easy to check that $K_{\lambda, T} \in C^{1}(H, \mathbb{R})$ and

$$
\begin{aligned}
\left\langle K_{\lambda, T}^{\prime}(u), v\right\rangle= & \langle u, v\rangle+\lambda D_{T}(u) \int_{\mathbb{R}_{3}} \phi_{u} u v \mathrm{~d} x+\frac{\lambda}{2 T^{2}} \eta^{\prime}\left(\frac{\|u\|^{2}}{T^{2}}\right)\langle u, v\rangle \int_{\mathbb{R}_{3}} \phi_{u} u^{2} \mathrm{~d} x \\
& -\int_{\mathbb{R}_{3}} f(u) v \mathrm{~d} x .
\end{aligned}
$$

In the following, we try to find a critical point $z_{\lambda}$ of $K_{\lambda, T}$ on $H$ for small $\lambda>0$. Then, by showing that $\left\|z_{\lambda}\right\| \leq T$, we will prove that $z_{\lambda}$ also solves the original problem (1.1). Similarly, we can define the Nehari manifold of $K_{\lambda, T}$ as

$$
\mathcal{N}_{\lambda, T}=\left\{u \in H \backslash\{0\},\left\langle K_{\lambda, T}^{\prime}(u), u\right\rangle=0\right\},
$$

and the nodal-Nehari manifold

$$
\mathcal{M}_{\lambda, T}=\left\{u \in H, u^{ \pm} \neq 0 \text { and }\left\langle K_{\lambda, T}^{\prime}(u), u^{ \pm}\right\rangle=0\right\} .
$$

What's more, we denote

$$
c_{\lambda, T}:=\inf _{u \in \mathcal{N}_{\lambda, T}} K_{\lambda, T}(u) \text { and } g_{\lambda, T}:=\inf _{u \in \mathcal{M}_{\lambda, T}} K_{\lambda, T}(u) .
$$

We have the following result.

Theorem 2.1. Assume that (f1)-(f4), (V1) and (V2) hold. Then there exists $\tilde{\lambda}>0$ such that for all $\lambda \in(0, \tilde{\lambda})$, the functional $K_{\lambda, T}$ possesses one least energy critical point $u_{\lambda} \in \mathcal{N}_{\lambda, T}$ which is constant sign and one least energy sign-changing critical point $z_{\lambda} \in \mathcal{M}_{\lambda, T}$. Moreover, the energy of the sign-changing critical point is strictly greater than the least energy, that is

$$
g_{\lambda, T}=K_{\lambda, T}\left(z_{\lambda}\right)>c_{\lambda, T}=K_{\lambda, T}\left(u_{\lambda}\right) .
$$

Lemma 2.1. For each $u \in H$ with $u^{ \pm} \neq 0$, there exists a pair $\left(t_{u}, s_{u}\right) \in \mathbb{R} \times \mathbb{R}$ with $t_{u}, s_{u}>0$ such that $t_{u} u^{+}+s_{u} u^{-} \in \mathcal{M}_{\lambda, T}$, moreover

$$
K_{\lambda, T}\left(t_{u} u^{+}+s_{u} u^{-}\right)=\max _{t, s \geq 0} K_{\lambda, T}\left(t u^{+}+s u^{-}\right) .
$$

Proof. For any $u \in H$ with $u^{ \pm} \neq 0$, define the function $\varphi:[0, \infty) \times[0, \infty) \rightarrow \mathbb{R}$ by

$$
\varphi(t, s):=K_{\lambda, T}\left(t u^{+}+s u^{-}\right)
$$

and its gradient is

$$
\begin{aligned}
\nabla \varphi(t, s) & =\left(\nabla \varphi_{1}(t, s), \nabla \varphi_{2}(t, s)\right)=\left(\frac{\partial \varphi}{\partial t}(t, s), \frac{\partial \varphi}{\partial s}(t, s)\right) \\
& =\left(\left\langle K_{\lambda, T}^{\prime}\left(t u^{+}+s u^{-}\right), u^{+}\right\rangle,\left\langle K_{\lambda, T}^{\prime}\left(t u^{+}+s u^{-}\right), u^{-}\right\rangle\right) .
\end{aligned}
$$

By (f1) and (f2), for any $\varepsilon>0$ and $p \in(2,6)$, there exists $C(\varepsilon)$ such that 


$$
|f(u)| \leq \varepsilon|u|+C(\varepsilon)|u|^{5} \text { and }|F(u)| \leq \frac{1}{2} \varepsilon|u|^{2}+\frac{C(\varepsilon)}{6}|u|^{6}, \quad \forall u \in \mathbb{R} .
$$

By (1.8), (2.6), the conclusion (i) of Lemma 1.1 and the property of $D_{T}$, we obtain

$$
\begin{aligned}
\varphi(t, s)= & \frac{1}{2} \int_{\mathbb{R}^{3}}\left[\left|\nabla\left(t u^{+}+s u^{-}\right)\right|^{2}+V(x)\left(t u^{+}+s u^{-}\right)^{2}\right] \mathrm{d} x \\
& +\frac{\lambda}{4} D_{T}\left(t u^{+}+s u^{-}\right) \int_{\mathbb{R}^{3}} \phi_{t u^{+}+s u^{-}}\left(t u^{+}+s u^{-}\right)^{2} \mathrm{~d} x-\int_{\mathbb{R}^{3}} F\left(t u^{+}+s u^{-}\right) \mathrm{d} x \\
\geq & \frac{1}{2}\left[\left\|t u^{+}\right\|^{2}+\left\|s u^{-}\right\|^{2}\right]-\int_{\mathbb{R}^{3}} F\left(t u^{+}+s u^{-}\right) \mathrm{d} x \\
\geq & \frac{t^{2}}{2}\left\|u^{+}\right\|^{2}+\frac{s^{2}}{2}\left\|u^{-}\right\|^{2}-\int_{\mathbb{R}^{3}} F\left(t u^{+}\right) \mathrm{d} x-\int_{\mathbb{R}^{3}} F\left(s u^{-}\right) \mathrm{d} x \\
\geq & \frac{t^{2}}{2}\left\|u^{+}\right\|^{2}+\frac{s^{2}}{2}\left\|u^{-}\right\|^{2}-\frac{\varepsilon t^{2}}{2}\left\|u^{+}\right\|_{2}^{2}-\frac{C(\varepsilon) t^{6}}{6}\left\|u^{+}\right\|_{6}^{6} \\
& -\frac{\varepsilon s^{2}}{2}\left\|u^{-}\right\|_{2}^{2}-\frac{C(\varepsilon) s^{6}}{6}\left\|u^{-}\right\|_{6}^{6} \\
\geq & \frac{t^{2}}{2}\left\|u^{+}\right\|^{2}+\frac{s^{2}}{2}\left\|u^{-}\right\|^{2}-C_{2}^{\prime} \frac{\varepsilon t^{2}}{2}\left\|u^{+}\right\|^{2}-C_{6}^{\prime} \frac{C(\varepsilon) t^{6}}{6}\left\|u^{+}\right\|^{6} \\
& -C_{2}^{\prime} \frac{\varepsilon s^{2}}{2}\left\|u^{-}\right\|^{2}-C_{6}^{\prime} \frac{C(\varepsilon) s^{6}}{6}\left\|u^{-}\right\|^{6},
\end{aligned}
$$

where every constant $C_{p}^{\prime}(p=2,6)$ is non-negative and $\varepsilon>0$. Then, for $(t, s)$ small enough, $\varphi(t, s)>0$. On the other hand, we can get that from (f3), for $t>0$ large enough, there exists a large $M>0$ such that

$$
|f(t)| \geq M|t| \text { and }|F(t)| \geq M|t|^{2} \text {. }
$$

Hence, for $(t, s)$ sufficiently large, from (2.7), we have

$$
\begin{aligned}
\varphi(t, s)= & K_{\lambda, T}\left(t u^{+}+s u^{-}\right) \\
= & \frac{1}{2}\left\|t u^{+}+s u^{-}\right\|^{2}+\frac{\lambda}{4} D_{T}\left(t u^{+}+s u^{-}\right) \int_{\mathbb{R}^{3}} \phi_{t u^{+}+s u^{-}}\left(t u^{+}+s u^{-}\right)^{2} \mathrm{~d} x \\
& -\int_{\mathbb{R}^{3}} F\left(t u^{+}+s u^{-}\right) \mathrm{d} x \\
= & \frac{1}{2}\left\|t u^{+}+s u^{-}\right\|^{2}-\int_{\mathbb{R}^{3}} F\left(t u^{+}+s u^{-}\right) \mathrm{d} x \\
= & \frac{t^{2}}{2}\left\|u^{+}\right\|^{2}+\frac{s^{2}}{2}\left\|u^{-}\right\|^{2}-\int_{\mathbb{R}^{3}} F\left(t u^{+}\right) \mathrm{d} x-\int_{\mathbb{R}^{3}} F\left(s u^{-}\right) \mathrm{d} x \\
\leq & \frac{t^{2}}{2}\left\|u^{+}\right\|^{2}+\frac{s^{2}}{2}\left\|u^{-}\right\|^{2}-\int_{\mathbb{R}^{3}} M\left|t u^{+}\right|^{2} \mathrm{~d} x-\int_{\mathbb{R}^{3}} M\left|s u^{-}\right|^{2} \mathrm{~d} x \\
= & \frac{t^{2}}{2}\left\|u^{+}\right\|^{2}+\frac{s^{2}}{2}\left\|u^{-}\right\|^{2}-\left.\left.M t^{2} \int_{\mathbb{R}^{3}}\right|^{+}\right|^{2} \mathrm{~d} x-M s^{2} \int_{\mathbb{R}^{3}}\left|u^{-}\right|^{2} \mathrm{~d} x .
\end{aligned}
$$

Therefore, we can get $\varphi(t, s) \rightarrow-\infty$ when $|(t, s)| \rightarrow+\infty$. We can infer that there is a pair of $\left(t_{u}, s_{u}\right) \in \mathbb{R}^{+} \times \mathbb{R}^{+}$such that

$$
\varphi\left(t_{u}, s_{u}\right)=\max _{t, s>0} \varphi(t, s) .
$$

Then, we prove that $t_{u}, s_{u}>0$. Without loss of genreality, we assume that $\left(0, s_{u}\right)$ is the maximum point of $\varphi(t, s)$. Hence, we have 


$$
\begin{aligned}
\frac{\partial \varphi\left(t, s_{u}\right)}{\partial t}= & t\left\|u^{+}\right\|^{2}+\lambda t D_{T}\left(t u^{+}+s_{u} u^{-}\right)\left[\int_{\mathbb{R}^{3}} t^{2} \phi_{u^{+}}\left|u^{+}\right|^{2} \mathrm{~d} x+\int_{\mathbb{R}^{3}} s^{2} \phi_{u^{-}}\left|u^{+}\right|^{2} \mathrm{~d} x\right] \\
& +\frac{\lambda t}{2 T^{2}} \eta^{\prime}\left(\frac{\left\|t u^{+}+s_{u} u^{-}\right\|^{2}}{T^{2}}\right)\left\|u^{+}\right\|^{2} \int_{\mathbb{R}^{3}} \phi_{t u^{+}+s_{u} u^{u}}\left|t u^{+}+s_{u} u^{-}\right|^{2} \mathrm{~d} x \\
& -\int_{\mathbb{R}^{3}} f\left(t u^{+}\right) u^{+} \mathrm{d} x \\
\geq & t\left\|u^{+}\right\|^{2}-C_{1} \frac{\lambda t}{T^{2}}\left\|u^{+}\right\|^{2}\left\|t u^{+}+s_{u} u^{-}\right\|^{4}-\int_{\mathbb{R}^{3}} f\left(t u^{+}\right) u^{+} \mathrm{d} x .
\end{aligned}
$$

From (f1), we can get $\frac{\partial \varphi\left(t, s_{u}\right)}{\partial t}>0$ for $\lambda, s$ small enough, which implies that $\varphi\left(t, s_{u}\right)$ is increasing for $t$ small. This contradicts with the fact that $\left(0, s_{u}\right)$ is the maximum point of $\varphi(t, s)$. Therefore, $\left(t_{u}, s_{u}\right)$ is a positive maximum point of $\varphi(t, s)$.

Finally, according to the definition of $\varphi$, we note that $t_{u} u^{+}+s_{u} u^{-} \in \mathcal{M}_{\lambda, T}$ is equivalent to $\varphi^{\prime}(t, s)=0$ for any $t, s>0$. Since the pair of $\left(t_{u}, s_{u}\right)$ is a positive maximum point of $\varphi(t, s)$, we observe that

$$
\left.\frac{\partial \varphi(t, s)}{\partial t}\right|_{\left(t_{u}, s_{u}\right)}=\left.\frac{\partial \varphi(t, s)}{\partial s}\right|_{\left(t_{u}, s_{u}\right)}=0,
$$

then,

$$
\left\langle K_{\lambda, T}^{\prime}\left(t_{u} u^{+}+s_{u} u^{-}\right), u^{+}\right\rangle=\left\langle K_{\lambda, T}^{\prime}\left(t_{u} u^{+}+s_{u} u^{-}\right), u^{-}\right\rangle=0,
$$

which implies that $t_{u} u^{+}+s_{u} u^{-} \in \mathcal{M}_{\lambda, T}$, because of $t_{u}, s_{u}>0$. This completes the proof.

Corollary 2.2. For each $u \in H \backslash\{0\}$, there exists a $t_{u} \in \mathbb{R}$ with $t_{u}>0$ such that $t_{u} u^{+} \in \mathcal{N}_{\lambda, T}$, moreover

$$
K_{\lambda, T}\left(t_{u} u^{+}\right)=\max _{t \geq 0} K_{\lambda, T}\left(t u^{+}\right) .
$$

Lemma 2.3. (see [28] [29]) Let $r>0$ and $p \in[2,6)$. If $\left\{u_{n}\right\}$ is bounded in $H$ and

$$
\limsup _{n \rightarrow \infty, y \in \mathbb{R}^{3}} \int_{B_{r}(y)}\left|u_{n}\right|^{p} \mathrm{~d} x=0,
$$

then we have $u_{n} \rightarrow 0$ in $L^{q}\left(\mathbb{R}^{3}\right)$ for $q \in(2,6)$.

Similar to [25], we have the following lemma.

Lemma 2.4. Assume that (f1)-(f4), (V1) and (V2) hold. Then, for any $u \in H$ with $\|u\|>2 T^{2}$, one has

$$
K_{\lambda, T}(u) \geq K_{\lambda, T}(t u)+\frac{1-t^{2}}{2}\left\langle K_{\lambda, T}^{\prime}(u), u\right\rangle, \forall t \geq 0 .
$$

Lemma 2.5. Let $\left\{u_{n}\right\} \subset \mathcal{N}_{\lambda, T}$ be a minimum sequence of $c_{\lambda, T}$, then $\left\{u_{n}\right\}$ is bounded in $H$.

Proof. We prove this lemma by contradiction. Set the unit normal vector of the level surface of the functional $\varphi$ is $v_{n}:=\frac{u_{n}}{\left\|u_{n}\right\|}$, and suppose $\left\|u_{n}\right\| \rightarrow \infty$ as 
$n \rightarrow \infty$. Therefore, we have $\left\|v_{n}\right\|=1$. Going to a subsequence if necessary, we may assume that

$$
\begin{gathered}
v_{n} \rightarrow v \text { in } H ; \\
v_{n} \rightarrow v \text { in } L^{p}\left(\mathbb{R}^{3}\right), \quad 2<p<6 ; \\
v_{n} \rightarrow v \text { a.e. in } \mathbb{R}^{3} .
\end{gathered}
$$

Hence, we're going to consider two cases: $v=0$ or $v \neq 0$.

Case (i). $v=0$. For $p \in[2,6)$, then

$$
\limsup _{n \rightarrow \infty, y \in \mathbb{R}^{3}} \int_{B_{r}(y)}\left|v_{n}\right|^{p} \mathrm{~d} x=0 .
$$

By Lemma 2.3 and (2.8), we have $v_{n} \rightarrow 0$ in $L^{q}\left(\mathbb{R}^{3}\right)$ for $q \in(2,6)$. Let $T_{1}=\sqrt{2\left(c_{\lambda, T}+T^{2}\right)}$. By (f1) and (f2), for any $\varepsilon>0$ and $p \in(2,6)$, there is $C(\varepsilon)>0$ such that

$$
\begin{aligned}
& |f(u)| \leq \varepsilon\left(|u|+|u|^{5}\right)+C(\varepsilon)|u|^{p-1} \\
& \text { and }|F(u)| \leq \varepsilon\left(\frac{1}{2}|u|^{2}+\frac{1}{6}|u|^{6}\right)+\frac{C(\varepsilon)}{p}|u|^{p}, \quad \forall u \in \mathbb{R}
\end{aligned}
$$

Then, by (1.8), (2.8), (2.9), Lemma 2.4 and the property of $D_{T}$, for $n$ sufficiently large such that $T_{1}^{2} /\left\|u_{n}\right\|^{2} \leq 1$, one has

$$
\begin{aligned}
c_{\lambda, T} & =K_{\lambda, T}\left(u_{n}\right)+o(1) \\
& \geq K_{\lambda, T}\left(T_{1} v_{n}\right)+\left(\frac{1}{2}-\frac{T_{1}^{2}}{2\left\|u_{n}\right\|^{2}}\right)\left\langle K_{\lambda, T}^{\prime}(u), u\right\rangle+o(1) \\
& =\frac{T_{1}^{2}}{2}\left\|v_{n}\right\|^{2}+\frac{\lambda T_{1}^{2}}{4} D_{T}\left(T_{1} v_{n}\right) \int_{\mathbb{R}^{3}} \phi_{T_{1} v_{n}}\left(v_{n}\right)^{2} \mathrm{~d} x-\int_{\mathbb{R}^{3}} F\left(T_{1} v_{n}\right) \mathrm{d} x+o(1) \\
& =\frac{T_{1}^{2}}{2}\left\|v_{n}\right\|^{2}-\int_{\mathbb{R}^{3}} F\left(T_{1} v_{n}\right) \mathrm{d} x+o(1) \\
& \geq \frac{T_{1}^{2}}{2}-\int_{\mathbb{R}^{3}}\left[\varepsilon\left(\frac{1}{2}\left|T_{1} v_{n}\right|^{2}+\frac{1}{6}\left|T_{1} v_{n}\right|^{6}\right)+\frac{C(\varepsilon)}{p}\left|T_{1} v_{n}\right|^{p}\right] \mathrm{d} x+o(1) \\
& \geq \frac{T_{1}^{2}}{2}-\varepsilon \frac{T_{1}^{2}}{2} C_{2}^{\prime}\left\|v_{n}\right\|^{2}-\varepsilon \frac{T_{1}^{6}}{6} C_{6}^{\prime}\left\|v_{n}\right\|^{6}-\frac{C(\varepsilon)}{p} T_{1}^{p} \int_{\mathbb{R}^{3}}\left|v_{n}\right|^{p} \mathrm{~d} x+o(1) \\
& =c_{\lambda, T}+T^{2}-C_{3} \varepsilon+o(1),
\end{aligned}
$$

which is a contradiction by the arbitrariness of $\varepsilon$.

Case (ii). $v \not \equiv 0$. There are $r, \delta>0$ and a sequence $\left\{y_{n}\right\} \subset \mathbb{R}^{3}$ such that

$$
\lim _{n \rightarrow \infty} \int_{B_{r}\left(y_{n}\right)}\left|v_{n}\right|^{p} \mathrm{~d} x \geq \delta>0 .
$$

Let $\Omega=\left\{x \in \mathbb{R}^{3}: v(x) \neq 0\right\}$. Hence, for $x \in \Omega$, one has $\left|u_{n}(x)\right| \rightarrow \infty$ as $n \rightarrow \infty$. From (f3) and Fatou's Lemma, we have

$$
\begin{aligned}
0 & \leq \lim _{n \rightarrow \infty} \frac{K_{\lambda, T}\left(u_{n}\right)}{\left\|u_{n}\right\|^{2}}=\lim _{n \rightarrow \infty} \frac{\frac{1}{2}\left\|u_{n}\right\|^{2}+\frac{\lambda}{4} D_{T}\left(u_{n}\right) \int_{\mathbb{R}^{3}} \phi_{u_{n}} u_{n}^{2} \mathrm{~d} x-\int_{\mathbb{R}^{3}} F\left(u_{n}\right) \mathrm{d} x}{\left\|u_{n}\right\|^{2}} \\
& =\frac{1}{2}+\lim _{n \rightarrow \infty} \frac{\lambda}{4} D_{T}\left(u_{n}\right) \int_{\mathbb{R}^{3}} \phi_{u_{n}}\left|v_{n}\right|^{2} \mathrm{~d} x-\lim _{n \rightarrow \infty} \int_{\mathbb{R}^{3}} \frac{F\left(u_{n}\right)}{\left\|u_{n}\right\|^{2}} d x
\end{aligned}
$$




$$
\begin{aligned}
& =\frac{1}{2}-\lim _{n \rightarrow \infty} \int_{\mathbb{R}^{3}} \frac{F\left(u_{n}\right)}{u_{n}^{2}}\left|v_{n}\right|^{2} \mathrm{~d} x \leq \frac{1}{2}-\liminf _{n \rightarrow \infty} \int_{\Omega} \frac{F\left(u_{n}\right)}{u_{n}^{2}}\left|v_{n}\right|^{2} \mathrm{~d} x \\
& \leq \frac{1}{2}-\int_{\Omega} \liminf _{n \rightarrow \infty} \frac{F\left(u_{n}\right)}{u_{n}^{2}}\left|v_{n}\right|^{2} \mathrm{~d} x=-\infty,
\end{aligned}
$$

a contradiction. Therefore, $\left\{u_{n}\right\} \subset H$ is bounded.

Corollary 2.6. Let $\left\{u_{n}\right\} \subset \mathcal{M}_{\lambda, T}$ be a minimizing sequence of $g_{\lambda, T}$, then $\left\{u_{n}\right\}$ is bounded in H. Hence, there exists a constant $a>0$ such that $\left\|u_{n}\right\| \leq a$.

Lemma 2.7. If $\lambda \in\left(0, \frac{T^{2}}{a^{4} C_{1}}\right)$, then $K_{\lambda, T}$ satisfies $(P S)_{m_{\lambda, T}}$ condition.

Proof. In view of Corollary 2.6, let $\left\{u_{n}\right\} \subset \mathcal{M}_{\lambda, T}$ be such that

$$
K_{\lambda, T}\left(u_{n}\right) \rightarrow g_{\lambda, T} \text {, as } n \rightarrow \infty .
$$

Then, $\left\{u_{n}\right\}$ is bounded in $H$. Since $u_{n} \in \mathcal{M}_{\lambda, T}$, we have

$$
\begin{aligned}
& \left\|u_{n}^{ \pm}\right\|^{2}+\lambda D_{T}\left(u_{n}\right) \int_{\mathbb{R}^{3}} \phi_{u_{n}}\left|u_{n}^{ \pm}\right|^{2} \mathrm{~d} x+\frac{\lambda}{2 T^{2}} \eta^{\prime}\left(\frac{\left\|u_{n}\right\|^{2}}{T^{2}}\right)\left\|u_{n}^{ \pm}\right\|^{2} \int_{\mathbb{R}^{3}} \phi_{u_{n}} u_{n}^{2} \mathrm{~d} x \\
& =\int_{\mathbb{R}^{3}} f\left(u_{n}^{ \pm}\right) u_{n}^{ \pm} \mathrm{d} x .
\end{aligned}
$$

Then, by (f1), (f2), (1.8) and (2.11), one has

$$
\begin{aligned}
\left(1-\frac{\lambda a^{4}}{T^{2}} C_{1}\right)\left\|u_{n}^{ \pm}\right\|^{2} & \leq\left\|u_{n}^{ \pm}\right\|^{2}-\frac{\lambda}{T^{2}}\left\|u_{n}^{ \pm}\right\|^{2} C_{1}\left\|u_{n}\right\|^{4} \\
& \leq\left\|u_{n}^{ \pm}\right\|^{2}+\frac{\lambda}{2 T^{2}} \eta^{\prime}\left(\frac{\left\|u_{n}\right\|^{2}}{T^{2}}\right)\left\|u_{n}^{ \pm}\right\|^{2} \int_{\mathbb{R}^{3}} \phi_{u_{n}} u_{n}^{2} \mathrm{~d} x \\
& \leq \varepsilon \int_{\mathbb{R}^{3}}\left|u_{n}^{ \pm}\right|^{2} \mathrm{~d} x+C(\varepsilon) \int_{\mathbb{R}^{3}}\left|u_{n}^{ \pm}\right|^{6} \mathrm{~d} x \\
& \leq \varepsilon C_{2}^{\prime}\left\|u_{n}^{ \pm}\right\|^{2}+C(\varepsilon) C_{6}^{\prime}\left\|u_{n}^{ \pm}\right\|^{6} .
\end{aligned}
$$

Therefore, there exists a constant $\rho>0$ such that

$$
\left\|u_{n}^{ \pm}\right\|^{2} \geq \rho, \quad \forall \lambda \in\left(0, \frac{T^{2}}{a^{4} C_{1}}\right) .
$$

Let $\left\{u_{n}\right\} \subset H$ be a $(P S)_{g_{\lambda, T}}$ sequence for $K_{\lambda, T}$, i.e.

$$
\lim _{n \rightarrow+\infty} K_{\lambda, T}\left(u_{n}\right)=g_{\lambda, T} \text { and } \lim _{n \rightarrow+\infty} K_{\lambda, T}^{\prime}\left(u_{n}\right)=0 .
$$

We can derive from Lemma 2.3 that $u_{n}$ is bounded in $H$, up to a subsequence, there exists $u \in H$ such that

$$
\begin{gathered}
u_{n} \rightarrow u \text { in } H ; \\
u_{n} \rightarrow u \text { in } L^{q}\left(\mathbb{R}^{3}\right), \quad 2<q<6 ; \\
u_{n} \rightarrow u \text { a.e. in } \mathbb{R}^{3} .
\end{gathered}
$$

From (1.8), (2.9), (2.15), Corollary 2.6 and Hölder inequality, we have

$$
\int_{\mathbb{R}^{3}} f\left(u_{n}\right)\left(u_{n}-u\right) \mathrm{d} x \rightarrow 0 \text { as } n \rightarrow \infty .
$$


From (1.11), (2.15), Lemma 1.1 and Hölder inequality, we obtain

$$
\begin{aligned}
\int_{\mathbb{R}^{3}} \phi_{u_{n}} u_{n}\left(u_{n}-u\right) \mathrm{d} x & \leq\left\|\phi_{u_{n}}\right\|_{6}\left\|u_{n}\right\|_{2}\left\|u_{n}-u\right\|_{3} \\
& \leq S^{-\frac{1}{2}}\left\|\phi_{u_{n}}\right\|_{\mathcal{D}^{1,2}}\left\|u_{n}\right\|_{2}\left\|u_{n}-u\right\|_{3} \\
& \leq C\left\|u_{n}\right\|^{2}\left\|u_{n}\right\|_{2}\left\|u_{n}-u\right\|_{3} \rightarrow 0 \text { as } n \rightarrow \infty .
\end{aligned}
$$

By (2.2), (2.14), (2.16), (2.17), for $n$ large enough, we have

$$
\begin{aligned}
o(1)= & \left\langle K_{\lambda, T}^{\prime}\left(u_{n}\right), u_{n}-u\right\rangle \\
= & \left\langle u_{n}, u_{n}-u\right\rangle+\lambda D_{T}\left(u_{n}\right) \int_{\mathbb{R}^{3}} \phi_{u_{n}} u_{n}\left(u_{n}-u\right) \mathrm{d} x \\
& +\frac{\lambda}{2 T^{2}} \eta^{\prime}\left(\frac{\left\|u_{n}\right\|^{2}}{T^{2}}\right)\left\langle u_{n}, u_{n}-u\right\rangle \int_{\mathbb{R}^{3}} \phi_{u_{n}} u_{n}^{2} \mathrm{~d} x-\int_{\mathbb{R}^{3}} f\left(u_{n}\right)\left(u_{n}-u\right) \mathrm{d} x \\
= & {\left[1+\frac{\lambda}{2 T^{2}} \eta^{\prime}\left(\frac{\left\|u_{n}\right\|^{2}}{T^{2}}\right) \int_{\mathbb{R}^{3}} \phi_{u_{n}} u_{n}^{2} \mathrm{~d} x\right]\left\langle u_{n}, u_{n}-u\right\rangle+o(1), }
\end{aligned}
$$

Hence, from (2.18), we have $\left\|u_{n}\right\| \rightarrow\|u\|$. The proof is completed.

Lemma 2.7. The $g_{\lambda, T}$ is achieved at some $z_{\lambda} \in \mathcal{M}_{\lambda, T}$ for $\lambda$ small, which is a critical point of $K_{\lambda, T}$ in $H$.

Proof. By Lemma 2.6, we know that $K_{\lambda, T}$ satisfies $(P S)_{m_{\lambda, T}}$ condition, then, there exists a $u \in H$ such that

$$
\begin{aligned}
& u_{n} \rightarrow u, \\
& u_{n}^{+} \rightarrow v, \\
& u_{n}^{-} \rightarrow w,
\end{aligned}
$$

in $H$ as $n \rightarrow \infty$. Then, by (2.12), one has $\left\|u_{n}^{-}\right\| \geq C_{4}>0$, likewise, $\left\|u_{n}^{+}\right\| \geq C_{5}>0$. It means that $v, w \neq 0$. Since $H$ is a Hilbert space and the project mapping $u \mapsto u^{ \pm}$is continuous in $H$, we get $u^{+}=v$ and $u^{-}=w$, then $u=u^{+}+u^{-}$is a sign-changing function. And then we show that $u \in \mathcal{M}_{\lambda, T}$. Since $u_{n} \in \mathcal{M}_{\lambda, T}$, one has

$$
\left\langle K_{\lambda, T}^{\prime}\left(u_{n}\right), u_{n}^{+}\right\rangle=\left\langle K_{\lambda, T}^{\prime}\left(u_{n}\right), u_{n}^{-}\right\rangle=0
$$

by (2.19) and passing to the limit, one has

$$
\left\langle K_{\lambda, T}^{\prime}(u), u^{+}\right\rangle=\left\langle K_{\lambda, T}^{\prime}(u), u^{-}\right\rangle=0,
$$

which means that $u \in \mathcal{M}_{\lambda, T}$ and $K_{\lambda, T}(u)=g_{\lambda, T}$. So the minimum value of $\left.K_{\lambda, T}\right|_{\mathcal{M}_{\lambda, T}}$ is achieved at $u$, therefore $u$ is a nontrivial critical point of $K_{\lambda, T}$ in $\mathcal{M}_{\lambda, T}$.

We also need to show that $u$ is the critical point of $K_{\lambda, T}$ in $H$. Because $u$ is a critical point of $K_{\lambda, T}$ in $\mathcal{M}_{\lambda, T}$, we have that $K_{\lambda, T}^{\prime}(u)=0$ in $\mathcal{M}_{\lambda, T}$. Then, there is a Lagrange multipiler $\varsigma$ such that

$$
K_{\lambda, T}^{\prime}(u)=\varsigma \chi^{\prime}(u)=0,
$$

where $\chi(u)=\left\langle K_{\lambda, T}^{\prime}(u), u\right\rangle$. That's enough to prove that $\varsigma=0$. By (2.20), one has 


$$
\left\langle K_{\lambda, T}^{\prime}(u), v\right\rangle-\varsigma\left\langle\chi^{\prime}(u), v\right\rangle=0, \text { for any } v \in H .
$$

Taking $v=u$, meanwhile, for any $u \in H$ with $\|u\|^{2}>2 T^{2}$, we calculated that

$$
\begin{aligned}
\left\langle\varsigma^{\prime}(u), u\right\rangle= & 2\|u\|^{2}+2 \lambda D_{T}(u) \int_{\mathbb{R}^{3}} \phi_{u} u^{2} \mathrm{~d} x+\lambda \eta^{\prime}\left(\frac{\|u\|^{2}}{T^{2}}\right) \frac{2\|u\|^{2}}{T^{2}} \int_{\mathbb{R}^{3}} \phi_{u} u^{2} \mathrm{~d} x \\
& +\frac{\lambda}{2 T^{2}} \eta^{\prime \prime}\left(\frac{\|u\|^{2}}{T^{2}}\right) \frac{2\|u\|^{2}}{T^{2}}\|u\|^{2} \int_{\mathbb{R}^{3}} \phi_{u} u^{2} \mathrm{~d} x+\frac{\lambda}{T^{2}} \eta^{\prime}\left(\frac{\|u\|^{2}}{T^{2}}\right)\|u\|^{2} \int_{\mathbb{R}^{3}} \phi_{u} u^{2} \mathrm{~d} x \\
& +\frac{\lambda}{T^{2}} \eta^{\prime}\left(\frac{\|u\|^{2}}{T^{2}}\right)\|u\|^{2} \int_{\mathbb{R}^{3}} \phi_{u} u^{2} \mathrm{~d} x-\left\{\int_{\mathbb{R}^{3}}\left[f^{\prime}(u) \cdot u^{2}+f(u) \cdot u\right] \mathrm{d} x\right\} \\
= & 2\|u\|^{2}+\lambda \int_{\mathbb{R}^{3}} \phi_{u} u^{2} \mathrm{~d} x\left[2 D_{T}+4 \eta^{\prime}\left(\frac{\|u\|^{2}}{T^{2}}\right) \frac{\|u\|^{2}}{T^{2}}+\frac{\|u\|^{4}}{T^{4}} \eta^{\prime \prime} \frac{\|u\|^{2}}{T^{2}}\right] \\
& -\int_{\mathbb{R}^{3}}\left[f^{\prime}(u) \cdot u^{2}-f(u) \cdot u\right] \mathrm{d} x \\
\leq & 2\|u\|^{2}+\lambda C_{1}\|u\|^{4}\left[4 \eta^{\prime}\left(\frac{\|u\|^{2}}{T^{2}}\right) \frac{\|u\|^{2}}{T^{2}}+\frac{\|u\|^{4}}{T^{4}} \eta^{\prime \prime}\left(\frac{\|u\|^{2}}{T^{2}}\right)\right] \\
& -\int_{\mathbb{R}^{3}}\left[f^{\prime}(u) \cdot u^{2}-f(u) \cdot u\right] \mathrm{d} x \\
\leq & 2\|u\|^{2}+\lambda C_{1}\|u\|^{4}\left[8 \eta^{\prime}\left(\frac{\|u\|^{2}}{T^{2}}\right)+4 \eta^{\prime \prime}\left(\frac{\|u\|^{2}}{T^{2}}\right)\right] \\
& -\int_{\mathbb{R}^{3}}\left[f^{\prime}(u) \cdot u^{2}-f(u) \cdot u\right] \mathrm{d} x .
\end{aligned}
$$

And we can find out from (f4) that there is a positive constant $\beta$ such that

$$
\int_{\mathbb{R}_{3}}\left[f^{\prime}(u) u^{2}-f(u) u\right] \mathrm{d} x \geq \beta>0 .
$$

Hence, $\left\langle\varsigma^{\prime}(u), u\right\rangle<0$ for $\lambda$ sufficiently small, which together with (2.21) shows that $\varsigma=0$. Thus, the proof is completed.

Corollary 2.8. The $c_{\lambda, T}$ is achieved at some $u \in \mathcal{N}_{\lambda, T}$, which is a critical of $K_{\lambda, T}$ in $H$.

Proof of Theorem 2.1. Through the lemmas and corollaries in this Section, we know that $K_{\lambda, T}$ has a least energy critical point and a least energy signchanging critical point, which are $u_{\lambda}$ and $z_{\lambda}$ respectively. For $z_{\lambda}^{+}$, by the above discussions, there exists a $t=t_{z_{\lambda}^{+}}$such that $t_{z_{\lambda}^{+}} z_{\lambda}^{+} \in \mathcal{N}_{\lambda, T}$, then

$$
\begin{aligned}
0 & <c_{\lambda, T}=K_{\lambda, T}\left(u_{\lambda}\right) \leq K_{\lambda, T}\left(t_{z_{\lambda}^{+}} z_{\lambda}^{+}\right)=K_{\lambda, T}\left(t_{z_{\lambda}^{+}} z_{\lambda}^{+}+0 z_{\lambda}^{-}\right) \\
& <K_{\lambda, T}\left(z_{\lambda}^{+}+z_{\lambda}^{-}\right)=K_{\lambda, T}\left(z_{\lambda}\right)=g_{\lambda, T} .
\end{aligned}
$$

Finally, we're going to prove that $u_{\lambda}$ is a constant sign. By assuming indirectly, assume that $u_{\lambda}$ is sign-changing, then $u_{\lambda} \in \mathcal{M}_{\lambda, T}$ and

$$
c_{\lambda, T}=K_{\lambda, T}\left(z_{\lambda}\right) \geq K_{\lambda, T}\left(z_{\lambda}\right)=g_{\lambda, T}>c_{\lambda, T},
$$

it is absurd. We've done the proof.

\section{Proof of the Main Result}

First, an important identity is given, which will be used to prove that $z_{\lambda}$ and 
$u_{\lambda}$ are uniformly bounded in $H$. For details of Pohožaev identity, one can see [30].

Lemma 3.1. If $u \in H$ is a critical point of $K_{\lambda, T}$, then for $\lambda>0$ small, $u$ satisfies

$$
\begin{aligned}
& \frac{1}{2} \int_{\mathbb{R}^{3}}|\nabla u|^{2} \mathrm{~d} x+\frac{1}{2} \int_{\mathbb{R}^{3}}[3 V(x)+\nabla V(x) x] u^{2} \mathrm{~d} x \\
& +\frac{5 \lambda}{4} D_{T}(u) \int_{\mathbb{R}^{3}} \phi_{u} u^{2} \mathrm{~d} x+\frac{3 \lambda}{T^{2}} \eta^{\prime}\left(\frac{\|u\|^{2}}{T^{2}}\right)\|u\|^{2} \int_{\mathbb{R}^{3}} \phi_{u} u^{2} \mathrm{~d} x \\
& =3 \int_{\mathbb{R}^{3}} F(u) \mathrm{d} x .
\end{aligned}
$$

Lemma 3.2. For $z_{\lambda}$ and $u_{\lambda}$ obtained in Theorem 2.1, if $T>0$ large enough and $\lambda>0$ small enough, then we have $\left\|z_{\lambda}\right\| \leq T$ and $\left\|u_{\lambda}\right\| \leq T$.

Proof. This part of proof is similar to [22]. However, it plays a key role in the proof of Theorem 1.1, so we give in detail here for completeness and convenience to the readers.

According to Hardy inequality [30], one has

$$
\|\nabla u\|_{2}^{2} \geq \frac{1}{4} \int_{\mathbb{R}^{3}} \frac{u^{2}}{|x|^{2}} \mathrm{~d} x, u \in H^{1}\left(\mathbb{R}^{3}\right) .
$$

Since $K_{\lambda, T}^{\prime}\left(z_{\lambda}\right)=0$, by Lemma 3.1, Lemma 1.1 (iv), (V2) and (3.1), one has

$$
\begin{aligned}
\int_{\mathbb{R}^{3}}\left|\nabla z_{\lambda}\right|^{2} \mathrm{~d} x= & 3 K_{\lambda, T}\left(z_{\lambda}\right)+\frac{\lambda}{2} D_{T}\left(z_{\lambda}\right) \int_{\mathbb{R}^{3}} \phi_{z_{\lambda}} z_{\lambda}^{2} \mathrm{~d} x \\
& +\frac{3 \lambda}{T^{2}} \eta^{\prime}\left(\frac{\left\|z_{\lambda}\right\|^{2}}{T^{2}}\right)\left\|z_{\lambda}\right\|^{2} \int_{\mathbb{R}^{3}} \phi_{z_{\lambda}} z_{\lambda}^{2} \mathrm{~d} x+\frac{1}{2} \int_{\mathbb{R}^{3}} \nabla V(x) \cdot x \cdot z_{\lambda}^{2} \mathrm{~d} x \\
\leq & 3 g_{\lambda, T}+\frac{\lambda}{2} D_{T}\left(z_{\lambda}\right) C_{1}\left\|z_{\lambda}\right\|^{4}+\frac{3 \lambda}{T^{2}}\left|\eta^{\prime}\left(\frac{\left\|z_{\lambda}\right\|^{2}}{T^{2}}\right)\right|\left\|z_{\lambda}\right\|^{6}+\theta\left\|\nabla z_{\lambda}\right\|_{2}^{2} \\
= & 3 g_{\lambda, T}+\frac{\lambda}{2} D_{T}\left(z_{\lambda}\right) C_{1}\left\|z_{\lambda}\right\|^{4}+\frac{3 \lambda}{T^{2}}\left|\eta^{\prime}\left(\frac{\left\|z_{\lambda}\right\|^{2}}{T^{2}}\right)\right|\left\|z_{\lambda}\right\|^{6}+\theta \int_{\mathbb{R}^{3}}\left|\nabla z_{\lambda}\right|^{2} \mathrm{~d} x,
\end{aligned}
$$

therefore, we have

$$
(1-\theta) \int_{\mathbb{R}^{3}}\left|\nabla z_{\lambda}\right|^{2} \mathrm{~d} x \leq 3 g_{\lambda, T}+\frac{\lambda}{2} D_{T}\left(z_{\lambda}\right) C_{1}\left\|z_{\lambda}\right\|^{4}+\frac{3 \lambda}{T^{2}}\left|\eta^{\prime}\left(\frac{\left\|z_{\lambda}\right\|^{2}}{T^{2}}\right)\right|\left\|z_{\lambda}\right\|^{6} .
$$

If $\left\|z_{\lambda}\right\|^{2} \geq 2 T^{2}$, then $D_{T}\left(z_{\lambda}\right)=0$. Therefore, the following inequality holds

$$
\int_{\mathbb{R}^{3}}\left|\nabla z_{\lambda}\right|^{2} \mathrm{~d} x \leq \frac{3}{1-\theta} g_{\lambda, T}+\frac{24}{1-\theta} \lambda C_{1}\left|\eta^{\prime}\left(\frac{\left\|z_{\lambda}\right\|^{2}}{T^{2}}\right)\right| T^{4}=C_{7}+C_{8} \lambda T^{4} .
$$

By (1.16), (2.9) and $\left\langle K_{\lambda, T}^{\prime}\left(z_{\lambda}\right), z_{\lambda}\right\rangle=0$, we have

$$
\begin{aligned}
& \left\|z_{\lambda}\right\|^{2}+\lambda D_{T}\left(z_{\lambda}\right) \int_{\mathbb{R}^{3}} \phi_{z_{\lambda}} z_{\lambda}^{2} \mathrm{~d} x+\frac{\lambda}{2 T^{2}} \eta^{\prime}\left(\frac{\left\|z_{\lambda}\right\|^{2}}{T^{2}}\right)\left\|z_{\lambda}\right\|^{2} \int_{\mathbb{R}^{3}} \phi_{z_{\lambda}} z_{\lambda}^{2} \mathrm{~d} x \\
& =\int_{\mathbb{R}^{3}} f\left(z_{\lambda}\right) z_{\lambda} \mathrm{d} x \leq \varepsilon \int_{\mathbb{R}^{3}}\left|z_{\lambda}\right|^{2} d x+C(\varepsilon) \int_{\mathbb{R}^{3}}\left|z_{\lambda}\right|^{6} \mathrm{~d} x .
\end{aligned}
$$


By $\mathcal{D}^{1,2}\left(\mathbb{R}^{3}\right) \hookrightarrow L^{6}\left(\mathbb{R}^{3}\right),(3.3)$ and $(\mathrm{V} 1)$, we have

$$
\begin{aligned}
\left(1-\frac{\varepsilon}{V_{0}}\right)\left\|z_{\lambda}\right\|^{2} & \leq\left(1-\frac{\varepsilon}{V(x)}\right)\left\|z_{\lambda}\right\|^{2} \\
& \leq C(\varepsilon) \int_{\mathbb{R}^{3}}\left|z_{\lambda}\right|^{6} \mathrm{~d} x-\frac{\lambda}{2 T^{2}} \eta^{\prime}\left(\frac{\left\|z_{\lambda}\right\|^{2}}{T^{2}}\right)\left\|z_{\lambda}\right\|^{2} \int_{\mathbb{R}^{3}} \phi_{z_{\lambda}} z_{\lambda}^{2} \mathrm{~d} x \\
& \leq C_{9} \lambda T^{4}+C_{10}\left(\int_{\mathbb{R}^{3}}\left|\nabla z_{\lambda}\right|^{2} \mathrm{~d} x\right)^{3} .
\end{aligned}
$$

Therefore, for $\varepsilon \leq \frac{V_{0}}{3}$, according to (3.2) and (3.4), we have

$$
\left\|z_{\lambda}\right\|^{2} \leq C_{11}\left(C_{7}+C_{8} \lambda T^{4}\right)^{3}+C_{12} \lambda T^{4} .
$$

We make the opposite hypothesis that $\left\|z_{\lambda}\right\|>T$, then, by (3.5), we have

$$
T^{2} \leq\left\|z_{\lambda}\right\|^{2} \leq C_{13}\left(1+\lambda T^{4}+\lambda^{2} T^{8}+\lambda^{3} T^{12}\right) .
$$

Choosing $T^{2}>\max \left\{1,4 C_{13}\right\}$ and $\lambda<\frac{1}{T^{4}}$, then (3.6) yields

$$
T^{2} \leq C_{13}\left(1+\lambda T^{4}+\lambda^{2} T^{8}+\lambda^{3} T^{12}\right)<4 C_{13},
$$

which is impossible. Thus $\left\|z_{\lambda}\right\| \leq T$, similarly, we can prove that $\left\|u_{\lambda}\right\| \leq T$. This completes the proof.

Proof of Theorem 1.1. Let $T$ be large enough and $\lambda$ small. We know from Theorem 2.1 that $K_{\lambda, T}$ has a least energy critical $u_{\lambda}$ at level $c_{\lambda, T}$ and a least energy sign-changing critical point $z_{\lambda}$ at level $g_{\lambda, T}$, and by Lemma 3.2 we have that $\left\|u_{\lambda}\right\| \leq T,\left\|z_{\lambda}\right\| \leq T$, therefore $K_{\lambda, T}=K_{\lambda}$ and $u_{\lambda}$ and $z_{\lambda}$ are critical points of $K_{\lambda}$ with $K_{\lambda}\left(u_{\lambda}\right)=c_{\lambda}$ and $K_{\lambda}\left(z_{\lambda}\right)=g_{\lambda}$. Hence, system (1.1) has a least energy sign-changing solution $z_{\lambda}$ and a ground state solution $u_{\lambda}$ which is constant sign. Moreover, since $K_{\lambda, T}=K_{\lambda}$, it follows from Lemma 2.1 that

$$
0 \leq c_{\lambda}=K_{\lambda}\left(u_{\lambda}\right)<K_{\lambda}\left(z_{\lambda}\right)=g_{\lambda}
$$

The proof is completed.

\section{Conclusion}

In this paper, we firstly proved that the Schrödinger-Poisson equation has a sign-changing solution by using a truncation technique, and then prove that the minimum sequence $\left\{u_{n}\right\}$ is bounded in $H$. What's more, according to the condition that $K_{\lambda, T}$ satisfies ( $P S$ ) sequence, we find out a critical point when the least energy sign-changing solution is achieved, and similarly find out a critical point when the ground state solution is achieved and prove that the sign-changing solution is strictly larger than the ground state solution. Finally, we prove that the critical points are uniformly bounded in $H$ using the Pohožaev identity. It is obviously that the truncation function has been successfully applied to solve the least energy sign-changing solution of the Schrödinger-Poisson system. We hope that the truncation technique can be widely used in the study of sign-changing 
solutions of similar systems.

\section{Acknowledgements}

The authors would like to thank the referees for their useful suggestions which have significantly improved the paper.

\section{Funding}

This work is supported by the National Natural Science Foundation of China (No. 11961014, No. 61563013) and Guangxi Natural Science Foundation (2021GXNSFAA196040, 2018GXNSFAA281021).

\section{Availability of Data and Materials}

No data were used to support to the work.

\section{Authors' Contributions}

The authors declare that the study was realized in collaboration with the same responsibility. All authors read and approved the final manuscript.

\section{Conflicts of Interest}

The authors declare no conflicts of interest regarding the publication of this paper.

\section{References}

[1] Benci, V. and Donato, F. (1998) An Eigenvalue Problem for the Schrödinger-Maxwell Equations. Topological Methods in Nonlinear Analysis, 11, 283-293. https://doi.org/10.12775/TMNA.1998.019

[2] D’Aprile, T. and Mugnai, D. (2004) Solitary Waves for Nonlinear Klein-GordonMaxwell and Schrödinger-Maxwell Equations. Proceedings of the Royal Society of Edinburgh Section A: Mathematics, 134, 893-906. https://doi.org/10.1017/S030821050000353X

[3] Sánchez, O. and Soler, J. (2004) Long-Time Dynamics of Schrödinger-Possion-Slater System. Journal of Statistical Physics, 114, 179-204. https://doi.org/10.1023/B:JOSS.0000003109.97208.53

[4] Ambrosetti, A. (2008) On Schrödinger-Poisson Systems. Milan Journal of Mathematics, 76, 257-274. https://doi.org/10.1007/s00032-008-0094-Z

[5] Ambrosetti, A. and Ruiz, D. (2008) Multiple Bound States for the Schrödinger-Poisson Problem. Communications in Contemporary Mathematics, 10, 391-404. https://doi.org/10.1142/S021919970800282X

[6] Ruiz, D. (2006) The Schrödinger-Poisson Equation under the Effect of a Nonlinear Local Term. Journal of Functional Analysis, 237, 655-674. https://doi.org/10.1016/j.jfa.2006.04.005

[7] Ruiz, D. (2010) On the Schrödinger-Poisson-Slater System Behavior of Minimizers Radial and Nonradial Cases. Archive for Rational Mechanics and Analysis, 198, 349-368. https://doi.org/10.1007/s00205-010-0299-5

[8] Azzollini, A., d'Avenia, P. and Pomponio, A. (2010) On the Schrödinger-Maxwell 
Equations under the Effect of a General Nonlinear Term. Annales de P Institut Henri Poincaré C, Analyse non linéaire, 27, 779-791.

https://doi.org/10.1016/j.anihpc.2009.11.012

[9] Coclite, G.M. (2002) A Multiplicity Result for the Linear Schrödinger-Maxwell Equations with Negative Potential. Annales Polonici Mathematici, 79, 21-30.

https://doi.org/10.4064/ap79-1-2

[10] Li, F.L. and Zhang, Q. (2013) Existence of Positive Solutions to the Schrödinger-Poisson System without Compactness Conditions. Journal of Mathematical Analysis and Applications, 401, 754-762. https://doi.org/10.1016/j.jmaa.2013.01.002

[11] Azzollini, A. and Pomponio, A. (2008) Ground State Solutions for the Nonlinear Schrödinger-Maxwell Equations. Journal of Mathematical Analysis and Applications, 345, 90-108. https://doi.org/10.1016/j.jmaa.2008.03.057

[12] Teresa, D.A. and Dimitri, M. (2004) Non-Existence Results for the Coupled KleinGordon-Maxwell Equations. Advanced Nonlinear Studies, 4, 307-322. https://doi.org/10.1515/ans-2004-0305

[13] Hiroaki, K. (2007) On the Existence of a Solution for Elliptic System Related to the Maxwell-Schrödinger Equations. Nonlinear Analysis, 67, 1445-1456.

https://doi.org/10.1016/j.na.2006.07.029

[14] David, R. (2006) The Schrödinger-Poisson Equation under the Effect of a Nonlinear Local Term. Journal of Functional Analysis, 237, 655-674.

https://doi.org/10.1016/j.jfa.2006.04.005

[15] Ji, H.W. (2017) Study on the Existence of Sign-Changing Solutions of Case Theory Based a Class of Differential Equations Boundary-Value Problems. Advances in Pure Mathematics, 7, 686-691. https://doi.org/10.4236/apm.2017.712042

[16] Wang, Z. and Zhou, H. (2015) Sign-Changing Solutions for the Nonlinear Schrödinger-Poisson System in $\mathbb{R}^{3}$. Calculus of Variations and Partial Differential Equations, 52, 927-943. https://doi.org/10.1007/s00526-014-0738-5

[17] Shuai, W. and Wang, Q.F. (2015) Existence and Asymptotic Behavior of SignChanging Solutions for the Nonlinear Schrödinger-Poisson System in $\mathbb{R}^{3}$. Zeitschrift für angewandte Mathematik und Physik, 66, 3267-3282.

https://doi.org/10.1007/s00033-015-0571-5

[18] Ling, Z., Xu, J. and Zhu, X. (2016) Revisit to Sign-Changing Solutions for the Nonlinear Schrödinger-Poisson System in $\mathbb{R}^{3}$. Journal of Mathematical Analysis and Applications, 435, 783-789. https://doi.org/10.1016/j.jmaa.2015.10.076

[19] Chen, S.T. and Tang, X.H. (2016) Ground State Sign-Changing Solutions for a Class of Schrödinger-Poisson System Type Problems in $\mathbb{R}^{3}$. Zeitschrift für angewandte Mathematik und Physik, 67, Article No. 102.

https://doi.org/10.1007/s00033-016-0695-2

[20] Alves, C.O., Souto, M.A.S. and Soares, S.H.M. (2017) A Sign-Changing Solution for the Schrödinger-Poisson Equation. Rocky Mountain Journal of Mathematics, 47, 1-25. https://doi.org/10.1216/RMJ-2017-47-1-1

[21] Qian, X.T. (2021) Ground State Sign-Changing Solutions for a Class of Nonlocal Problem. Journal of Mathematical Analysis and Applications, 495, Article ID: 124753. https://doi.org/10.1016/j.jmaa.2020.124753

[22] Zhang, J. (2012) On the Schrödinger-Poisson System with a General Nonlinearity in the Critical Growth. Nonlinear Analysis, 75, 6391-6401. https://doi.org/10.1016/j.na.2012.07.008

[23] Jeanjean, L. and Coz, S.L. (2006) An Existence Stability Result for Standing Waves 
of Nonlinear Schrödinger Equations. Advances in Differential Equations, 11, 813-840.

[24] Li, Y.H., Li, F.Y. and Shi, J.P. (2012) Existence of a Positive Solution to Kirchhoff Type Problems without Compactness Conditions. Journal of Differential Equations, 253, 2285-2294. https://doi.org/10.1016/j.jde.2012.05.017

[25] Khoutir, S. (2021) Least Energy Sign-Changing Solutions for Super-Quadratic Schrödinger-Poisson Systems in $\mathbb{R}^{3}$. Journal of Applied Analysis \& Computation, 11, 1520-1534. https://doi.org/10.11948/20200274

[26] Willem, M. (1996) Minimax Theorems. Birkhäuser Boston Inc., Boston. https://doi.org/10.1007/978-1-4612-4146-1

[27] Cerami, G. and Vaira, G. (2010) Positive Solutions for Some Non-Autonomous Schrödinger-Poisson Systems. Journal of Differential Equations, 248, 521-543. https://doi.org/10.1016/j.jde.2009.06.017

[28] Lions, P.L. (1984) The Concentration-Compactness Principle in the Calculus of Variations. The Locally Compact Case. Part I. Annales de IInstitut Henri Poincaré C, Analyse non linéaire, 1, 109-145. https://doi.org/10.1016/S0294-1449(16)30428-0

[29] Lion, P.L. (1984) The Concentration-Compactness Principle in the Calculus of Variations. The Locally Compact Case. Part II. Annales de I Institut Henri Poincaré C, Analyse non linéaire, 1, 223-283. https://doi.org/10.1016/S0294-1449(16)30422-X

[30] Chen, S.T. and Tang, X.H. (2020) Berestycki-Lions Conditions on Ground State Solutions for a Nonlinear Schrödinger Equation with Variable Potentials. Advances in Nonlinear Analysis, 9, 496-515. https://doi.org/10.1515/anona-2020-0011 